\title{
Osteoprotegerina y Sistema RANKL/RANK: ¿el futuro del metabolismo óseo?
}

Ferrer Cañabate J, Tovar I, Martínez P. Osteoprotegerina y Sistema RANKL/RANK: ¿el futuro del metabolismo óseo? An Med Interna (Madrid) 2002; 19: 385-388.

El avance en los estudios moleculares y la incesante búsqueda de nuevas opciones terapéuticas nos sitúan en el comienzo de una nueva era en el estudio y entendimiento del metabolismo y remodelado del tejido óseo. La salud de nuestros huesos es consecuencia de los procesos de remodelado que en ellos se suceden a lo largo de toda la vida, encabezados por una controlada y permanente etapa de reabsorción llevada a cabo por los osteoclastos, y seguida en un perfecto equilibrio por una etapa de formación protagonizada por los osteoblastos. Cualquier desequilibrio en este balance resorción-formación conduce a una pérdida de tejido óseo si la resorción excede a la formación (osteoporosis, osteopenia,...), o a un exceso del mismo, y no por ello con una mayor calidad, si es la formación la que supera en gran medida a la resorción (osteopetrosis,...). El control de esta homeostasis parece estar influenciado por una gran número de citokinas, hormonas y factores de crecimiento, tanto en la puesta en escena de nuestros dos principales protagonistas, osteoblastos y osteoclastos, por diferenciación a partir de sus precursores inmaduros, como en cualquiera de las dos etapas del remodelado (1-7).

Los osteoblastos derivan de células madre mesenquimales, y los osteoclastos son el resultado de la diferenciación de sus precursores a partir de progenitores hematopoyéticos y su posterior evolución hasta osteoclastos maduros multinucleados (osteoclastogénesis). Ambos procesos dependen de varios factores puestos en su debido momento en juego, y en concreto, haciendo referencia al segundo, son de gran importancia los producidos tanto por los osteoblastos como por las células madre pluripotenciales de la médula ósea. Nos encontrábamos por tanto ante una interesante paradoja (8): la mayoría de los receptores para estas hormonas, citokinas y factores de crecimiento se encuentran mayormente en los osteoblastos, independientemente de si su activación desencadenaba procesos de formación o de resorción. Y más aún, nos hace plantearnos una segunda cuestión: si al parecer los osteoblastos juegan un papel importante en el desarrollo de los osteoclastos, ¿hasta dónde llega este papel, si los segundos van a ser los encargados de destruir la obra creada por los primeros?

Todo el mundo parece tener clara la importancia de la regulación de la osteoclastogénesis, pero lo que hasta ahora parecía no estar tan claro era quién o quiénes la llevaban a cabo, o al menos, quiénes eran los responsables que, hasta ahora anónimos, controlaban todo el proceso. El descubrimiento del sistema OPG/RANKL/RANK aporta muchísima luz no sólo al conocimiento de la fisiopatología ósea, sino que abre una nueva vía de estudio de posibles herramientas terapéuticas en la lucha frente a las enfermedades óseas.

El primero y quizás más importante de nuestros protagonistas es la osteoprotegerina (OPG: "to protect bone"), también conocida como factor de inhibición de la osteoclastogénesis (OCIF) o como TNFRSF11B. Es un nuevo miembro de la superfamilia de los receptores del factor de necrosis tumoral (TNFR), que a diferencia de todos sus parientes, no permanece tras su síntesis como una proteína transmembrana con el cometido de elaborar señales de transducción entre distintas células, sino que es secretada y no permanece anclada en membrana. Codificada por un gen situado en el cromosoma 8q23-24, fue descubierta simultáneamente pero de manera totalmente distinta por dos grupos de investigación, uno de ellos (Amgen, Inc. Group, USA) mientras estudiaba cDNAs en intestino de rata (9), y otro (Snow Brand Milk Group, Japon) mientras buscaban factores de inhibición y/o estimulación de los osteoclastos (10), aunque en este último caso ya Rodan y Martin en 1981 (11) planteaban la importancia del papel de los precursores de los osteoblastos (responsables de la producción de la OPG) en el control de la osteoclastogénesis. Una vez descubierta la OPG, numerosos estudios se sucedieron para desenmascarar y caracterizar tanto su estructura como sus funciones. Se sintetiza inicialmente como un propéptido de 401 aminoácidos, y tras la pérdida de un fragmento de 21 aminoácidos, queda como proteína madura con 380 aas $(9,10)$, momento a partir del cual pierde sus dominios transmembrana y citoplasmáticos y es secretada como proteína soluble. Su extremo N-terminal contiene 4 dominios ricos en cisteína (D1-D4) y es el más estrechamente relacionado con el receptor-2 de TNF y con el CD40. El extremo C-terminal posibilita la homodimerización de la molécula. $(9,10,12)$. El RNAm de la OPG se expresa en numerosos tejidos humanos (pulmón, corazón, riñones, hígado, intestino, estómago, cerebro, glándula tiroides y médula 
espinal) además de en el hueso $(9,10)$, en el cual su principal función parece ser la inhibición de la maduración de los osteoclastos y de su activación, tanto in vivo como in vitro (13). Esto queda claramente de manifiesto en los experimentos realizados con ratones $O P G$-knock out $(14,15)$, los cuales, aún de apariencia normal al nacimiento, tenían una mayor tasa de mortalidad en la adolescencia debida a un incremento en la incidencia de fracturas vertebrales y femorales. Sus tejidos óseos eran característicos de sujetos con un alto grado de remodelado: huesos con pérdida trabecular y porosidad cortical aumentada. Y algo más sorprendente aún: estos ratones OPG-knock out mostraban calcificaciones de la aorta y de las arterias renales ya desde las dos semanas de vida (14), calcificaciones que se encontraban en $2 / 3$ partes del animal ya a los dos meses.

Una vez descubierta la OPG, los siguientes actores principales fueron apareciendo uno tras otro. En segundo lugar apareció su ligando $(13,16)$ : OPG-L, también conocido como ODF (factor de diferenciación de los osteoclastos), y que resultó ser idéntico a dos miembros ya conocidos de la familia de los ligandos de TNF: el TRANCE (TNF-related activation induced citokine), inducido por activación de receptores de células T, y el RANKL (ligando de unión al Receptor Activador de NF-kB), un factor que se sabía estimulaba a células dendríticas $(17,18)$. OPG-L, codificado por un gen situado en el cromosoma $13 \mathrm{q} 14(17,18)$, aparece bien anclado a membrana de osteoblastos, células del estroma, células inmaduras mesenquimales de los bordes del cartílago y condrocitos hipertróficos, o bien es liberado de la superficie celular en forma de moléculas homotrímeras solubles por acción de metaloproteasas (19). Su RNAm se expresa no sólo en tejido óseo sino en médula ósea y tejidos linfáticos $(13,16-18)$. Su principal papel en el hueso es la estimulación de la diferenciación de los osteoclastos $(13,20)$, su activación (13) y la inhibición de su apoptosis (21). Junto al factor estimulante de colonias de macrófagos (M-CSF) son los dos factores necesarios y suficientes para completar todo el ciclo de maduración de los osteoclastos a partir de sus precursores inmaduros $(13,16)$. Como cabría esperar, los ratones RANKL-knock out padecían osteopetrosis severa, acompañada de no sólo defectos en la erupción dental (18) sino también de una falta total de nódulos linfáticos, defectos en la diferenciación de células T y B, y fallos en la formación de estructuras lobulo-alveolares mamarias durante el embarazo (22).

El tercero en discordia no tardó en aparecer (18). El receptor RANK (Receptor Activador de NF-kB), codificado por un gen del cromosoma 18q22.1 (18) y cuyo RNAm se expresa en osteoclastos maduros y en sus progenitores aislados de médula ósea (21), es un péptido de 616 aas que se encuentra principalmente en células de la estirpe monocítica-macrófaga, preosteoclastos, células $\mathrm{T}$ y $\mathrm{B}$, células dendríticas y fibroblastos $(18,19)$. Su activación conlleva no sólo una reorganización en el citoesqueleto del osteoclasto y cambios fundamentales para su activación, movilidad y establecimiento en la superficie ósea a resorber (23), sino que desencadena una señal de supervivencia en el osteoclasto maduro (24). Si por tanto, tal y como se pensaba, conseguíamos tener ratones deficientes en este receptor RANK, las consecuencias eran previsibles: severa osteopetrosis, con características radiológicas e histológicas del tejido óseo casi iguales a las de los ratones RANK-L knock out $(25,26)$. Además, no sólo había también una ausencia total de osteoclastos y de nódulos linfáticos, sino que los ratones desarrollaban hipocalcemia e hipofosfatemia, con marcados niveles de parathormona (25).

Todos los datos reunidos hasta este momento evidenciaban claramente que nos encontrábamos ante un sistema regulador importantísimo del metabolismo óseo, con un equilibrio muy estrecho entre los procesos de formación y resorción. La interacción entre OPGL liberado por los osteoblastos y/o células del estroma, y su receptor RANK expresado en los precursores osteoclásticos, y para la cual la OPG actúa como receptor señuelo secuestrando a OPGL y evitando la activación de RANK, es fundamental para un correcto desarrollo de la osteoclastogénesis, no sólo para la diferenciación de los precursores hematopoyéticos hasta osteoclastos maduros, sino también para la fusión de los osteoclastos mononucleares comprometidos entre sí para formar osteoclastos multinucleados, así como para activar a los osteoclastos maduros (Fig. 1). Aún existiendo una vía alternativa para la regulación de la osteoclastogénesis distinta y paralela a la de la activación de RANK, y que parece estar mediada por una activación minoritaria de NF-kB en la que juegan un papel muy importante tanto IL-6 como la activación de gp130 (27), ésta segunda vía es mucho menos importante y un afectación en la mayoritaria conduce a serios trastornos en el metabolismo de la masa ósea.

Llegados a este punto, parece evidente que la comprensión de todo este complejo sistema OPG/RANKL/ RANK es fundamental para comprender toda la fisiopatología ósea. Podríamos ya incluso responder a la primera pregunta que nos formulábamos, pues se ha comprobado que los osteoblastos maduros producen cantidades importantes de OPG, que bloquearía el transcurso de la osteoclastogénesis e impediría la formación de osteoclastos, auténticos destructores de su artesonado, mientras que los osteoblastos inmaduros mediante descensos en la expresión de OPG e incrementos en la de OPGL (28) son capaces de reclutar a los osteoclastos que limpiarán de tejido óseo viejo su lugar de trabajo. Pero aún hemos de ir más allá, porque las repercusiones de todo este descubrimiento no se quedan aquí. Si la regulación de todo el remodelado óseo se ve seriamente influenciada por este sistema, parece lógico que muchas de las patologías óseas que conocemos puedan tener en su génesis trastornos en el mismo. Y más aún, podríamos incluso llegar a plantearnos la posibilidad de emplear OPG como una alternativa terapéutica en aquellos casos en los que quisiésemos bloquear el proceso de resorción de una manera "artificial".

Recientes estudios han demostrado que los estrógenos (29) y TGK-b (30,31), un factor de crecimiento inducido en los osteoblastos por los estrógenos, incrementan la producción de OPG por los osteoblastos y células del estroma, lo cual neutralizaría a OPG-L y controlaría en gran medida la osteoclastogénesis. Si tras la menopausia la mujer se encuentra en un estado de deprivación estrogénica, perdería esta capacidad reguladora y el aumento de la actividad RANKLRANK parecería ser la responsable de la pérdida ósea que se experimenta en postmenopausia. Yano et al (32) han demostrado que la concentración de OPG en suero aumenta con la edad, y que las mujeres postmenopáusicas osteoporóticas tienen, pese a lo que cabría esperar, niveles de OPG algo mayores que las no osteoporóticas, lo cual concluyen podría ser reflejo o consecuencia de un efecto compensatorio orgánico en respuesta al aumento de la resorción ósea en estos sujetos. Sin embargo, Arrighi y cols. (33) no encuentran ese aumento 


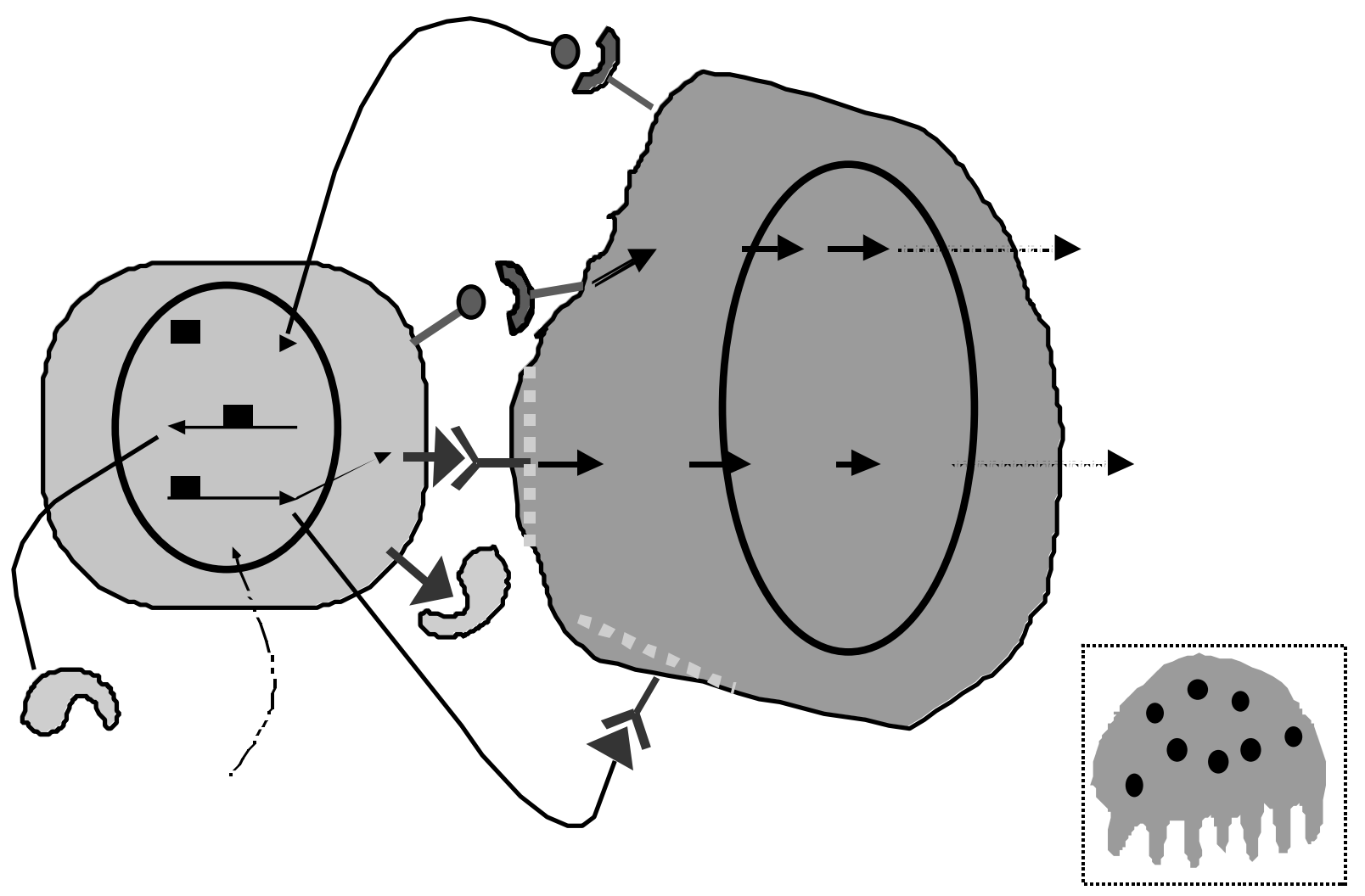

Fig. 1. Sistema OPG/RANK-L/RANK.

de OPG paralelo a la edad. Es de destacar el trabajo de Bekker et al (34), que ya orientan el posible empleo en terapia en humanos de la OPG Ya se sabía que en ratas ovariectemizadas la OPG no sólo prevenía la pérdida ósea sino que incrementaba el volumen óseo (9), y demuestran que una simple dosis subcutánea en mujeres osteoporóticas postmenopáusicas no sólo disminuye los niveles de uNTX ya a las 12 horas tras la administración, sino que los mantiene disminuidos durante un mes sin que se presenten efectos secundarios. Podemos encontrarnos, pues, ante un tratamiento efectivo y seguro para la osteoporosis postmenopáusica (8).

Los niveles elevados de la PTH aumentan RANKL y disminuyen la expresión de OPG por los osteoblastos, produciendo un efecto catabólico en el hueso (35). Sin embargo, concentraciones de PTH intermitentes no parecen alterar el ratio OPG/RANKL (36), lo cual podría explicar la disparidad de efectos de la parathormona (anabolismo o catabolismo) sobre el hueso en función de su pauta de administración (37).

En la enfermedad de Paget (donde RANKL está sobrexpresado y las células preosteoclásticas de las zonas lesionadas tienen una mayor afinidad por él (38)), al igual que en la osteolisis familiar, encontramos mutaciones en la región codificadora del péptido señal de la proteína RANK (39), las cuales conducen a la producción de unos receptores RANK defectuosos y por tanto a una posible malfunción de todo este sistema regulador.

Podíamos seguir mucho más allá aún, pues la gran cantidad de estudios que en sólo dos años se están desarrollando en torno al sistema OPG/RANKL/RANK no parecen querer limitarse a osteoporosis, Paget $u$ otras alteraciones óseas. La activa- ción de los linfocitos $\mathrm{T}$ (como tiene lugar por ejemplo en la artritis reumatoide) conlleva un incremento en la expresión de RANKL (40), el cual en presencia de M-CSF parece poder inducir la diferenciación de los macrófagos sinoviales hasta osteoclastos (41), lo cual puede ser la causa de la pérdida ósea en la zona periarticular de la lesiones artríticas inflamatorias. Podríamos encontrarnos pues no ante una explicación molecular sólo de la pérdida ósea en la artritis reumatoide, sino ante la causa del balance óseo negativo que tiene lugar en muchas enfermedades en las que el sistema inmune juega un papel preponderante, tales como las leucemias en niños o en adultos, las metástasis cancerosas (el papel de la OPG en el diagnóstico de metástasis óseas en pacientes con carcinoma de próstata parece ser incluso mejor que el de la isoenzima ósea de la fosfatasa alcalina) (42), las enfermedades autoinmunes o distintas infecciones virales, casos todos éstos que nos abren una nueva vía de estudio más: la del papel de los linfocitos T como reguladores del metabolismo óseo (43).

Todas estas razones y las que con seguridad seguirán apareciendo en próximas fechas parecen situarnos, y ojalá no nos equivoquemos, ante uno de los hallazgos más importantes en el campo de la biología, y muy probablemente, ante una línea terapéutica que, si las jugarretas del entramado molecular y celular no impiden, dará mucho que hablar.

\section{J. FERRER CAÑABATE, I. TOVAR, P. MARTÍNEZ}

Servicio de Análisis Clínicos. Hospital Universitario Virgen de la Arrixaca. Murcia 


\section{Bibliografía}

1. Suda T, Takahashi N, Martin TJ. Modulation of osteoclast differentiation. Endocr Rev 1992; 13 (1): 66-80.

2. Suda T, Nakamura I, Jimi E, Takahashi N. Regulation of osteoclast function. J Bone Miner Res 1997;12 (6): 869-79.

3. Roodman GD. Advances in bone biology: the osteoclast. Endocr Rev 1996; 17 (4): 308-32.

4. Reddy SV, Roodman GD. Control of osteoclast differentiation. Crit Rev Eukaryot Gene Expr 1998; 8 (1): 1-17.

5. Pacifici R. Estrogen, cytokines, and pathogenesis of postmenopausal osteoporosis. J Bone Miner Res 1996; 11 (8): 1043-51.

6. Manolagas SC, Jilka RL. Bone marrow, cytokines, and bone remodeling. Emerging insights into the pathophysiology of osteoporosis. N Engl J Med 1995; 332 (5): 305-11.

7. Jilka RL. Cytokines, bone remodeling, and estrogen deficiency: a 1998 update. Bone 1998; 23 (2): 75-81.

8. Kostenuik PJ, Shalhoub V. Osteoprotegerin: a physiological and pharmacological inhibitor of bone resorption. Curr Pharm Des 2001; 7 (8): 613-35.

9. Simonet WS, Lacey DL, Dunstan CR, Kelley M, Chang MS, Luthy R et al. Osteoprotegerin: a novel secreted protein involved in the regulation of bone density. Cell 1997; 89 (2): 309-19.

10. Yasuda H, Shima N, Nakagawa N, Mochizuki SI, Yano K, Fujise N et al. Identity of osteoclastogenesis inhibitory factor (OCIF) and osteoprotegerin (OPG): a mechanism by which OPG/OCIF inhibits osteoclastogenesis in vitro. Endocrinology 1998; 139 (3): 1329-37.

11. Rodan GA, Martin TJ. Role of osteoblasts in hormonal control of bone resorption - a hypothesis. Calcif Tissue Int 1982; 34 (3): 311.

12. Yamaguchi K, Kinosaki M, Goto M, Kobayashi F, Tsuda E, Morinaga T, Higashio K. Characterization of structural domains of human osteoclastogenesis inhibitory factor. J Biol Chem 1998; 273 (9): 5117-23.

13. Lacey DL, Timms E, Tan HL, Kelley MJ, Dunstan CR, Burgess T, et al. Osteoprotegerin ligand is a cytokine that regulates osteoclast differentiation and activation. Cell 1998; 93 (2): 165-76.

14. Bucay N, Sarosi I, Dunstan CR, Morony S, Tarpley J, Capparelli C et al. Osteoprotegerin-deficient mice develop early onset osteoporosis and arterial calcification. Genes Dev 1998; 12 (9): 1260-8.

15. Mizuno A, Amizuka N, Irie K, Murakami A, Fujise N, Kanno T, et al. Severe osteoporosis in mice lacking osteoclastogenesis inhibitory factor/osteoprotegerin. Biochem Biophys Res Commun 1998; 247 (3): 610-5.

16. Yasuda H, Shima N, Nakagawa N, Yamaguchi K, Kinosaki M, Mochizuki S, et al. Osteoclast differentiation factor is a ligand for osteoprotegerin/osteoclastogenesis-inhibitory factor and is identical to TRANCE/RANKL. Proc Natl Acad Sci USA 1998; 95 (7): 3597-602.

17. Wong BR, Rho J, Arron J, Robinson E, Orlinick J, Chao M, et al. TRANCE is a novel ligand of the tumor necrosis factor receptor family that activates c-Jun N-terminal kinase in T cells. J Biol Chem 1997; 272 (40): 25190-4.

18. Anderson DM, Maraskovsky E, Billingsley WL, Dougall WC, Tometsko ME, Roux ER, et al. A homologue of the TNF receptor and its ligand enhance T-cell growth and dendritic-cell function. Nature 1997; 390 (6656): 175-9.

19. Lum L, Wong BR, Josien R, Becherer JD, Erdjument-Bromage H, Schlondorff J. et al. Evidence for a role of a tumor necrosis factor-alpha (TNF-alpha)-converting enzyme-like protease in shedding of TRANCE, a TNF family member involved in osteoclastogenesis and dendritic cell survival. J Biol Chem 1999; 274 (19): 13613-8.

20. Malyankar UM, Scatena M, Suchland KL, Yun TJ, Clark EA, Giachelli CM. Osteoprotegerin is an alpha vbeta 3-induced, NF-kappa B-dependent survival factor for endothelial cells. J Biol Chem 2000; 275 (28): 20959-62.

21. Fuller K, Wong B, Fox S, Choi Y, Chambers TJ. TRANCE is necessary and sufficient for osteoblast-mediated activation of bone resorption in osteoclasts. J Exp Med 1998;188 (5): 997-1001.

22. Kong YY, Yoshida H, Sarosi I, Tan HL, Timms E, Capparelli C, et al. OPGL is a key regulator of osteoclastogenesis, lymphocyte development and lymph-node organogenesis. Nature 1999; 28; 397 (6717): 315-23.
23. Burgess TL, Qian Y, Kaufman S, Ring BD, Van G, Capparelli C et al. The ligand for osteoprotegerin (OPGL) directly activates mature osteoclasts. J Cell Biol 1999; 145 (3): 527-38.

24. Wong BR, Besser D, Kim N, Arron JR, Vologodskaia M et al. TRAN$\mathrm{CE}$, a TNF family member, activates Akt/PKB through a signaling complex involving TRAF6 and c-Src. Mol Cell. 1999; 4 (6): 1041-9.

25. Li J, Sarosi I, Yan XQ, Morony S, Capparelli C, Tan HL et al. RANK is the intrinsic hematopoietic cell surface receptor that controls osteoclastogenesis and regulation of bone mass and calcium metabolism. Proc Natl Acad Sci USA 2000; 97 (4): 1566-71.

26. Dougall WC, Glaccum M, Charrier K, Rohrbach K, Brasel K, De Smedt $\mathrm{T}$ et al. RANK is essential for osteoclast and lymph node development. Genes Dev 1999; 13 (18): 2412-24.

27. Hing L, Carlson L, Siebenlist U\&Boyce BF. Mechanism by which NF$\mathrm{kB}$ regulates osteoclast numbers. Bone 1998; (Supl. 1) S190 (Abstract no. 1172)

28. Nagai M, Sato N. Reciprocal gene expression of osteoclastogenesis inhibitory factor and osteoclast differentiation factor regulates osteoclast formation. Biochem Biophys Res Commun 1999; 257 (3): 719-23.

29. Hofbauer LC, Khosla S, Dunstan CR, Lacey DL, Spelsberg TC, Riggs BL. Estrogen stimulates gene expression and protein production of osteoprotegerin in human osteoblastic cells. Endocrinology 1999; 140 (9): 4367-70.

30. Murakami T, Yamamoto M, Ono K, Nishikawa M, Nagata N, et al. Transforming growth factor-beta1 increases mRNA levels of osteoclastogenesis inhibitory factor in osteoblastic/stromal cells and inhibits the survival of murine osteoclast-like cells. Biochem Biophys Res Commun 1998; 252 (3): 747-52.

31. Takai H, Kanematsu M, Yano K, Tsuda E, Higashio K, et al. Transforming growth factor-beta stimulates the production of osteoprotegerin/osteoclastogenesis inhibitory factor by bone marrow stromal cells. J Biol Chem 1998; 273 (42): 27091-6.

32. Yano K, Tsuda E, Washida N, Kobayashi F, Goto M, et al. Immunological characterization of circulating osteoprotegerin/osteoclastogenesis inhibitory factor: increased serum concentrations in postmenopausal women with osteoporosis. J Bone Miner Res 1999;14 (4): 518-27.

33. Arrighi HM, Hsieh A, Wong H, Khoo H, Reyes A, Asbury D et al. Osteoprotegerin serum levels in healthy volunteers. Bone 1998; (Supl. 1) S298 (Abstract no. T411)

34. Bekker PJ, Holloway D, Nakanishi A, Arrighi M, Leese PT, Dunstan $\mathrm{CR}$. The effect of a single dose of osteoprotegerin in postmenopausal women. J Bone Miner Res 2001; 16 (2): 348-60.

35. Lee SK, Lorenzo JA. Parathyroid hormone stimulates TRANCE and inhibits osteoprotegerin messenger ribonucleic acid expression in murine bone marrow cultures: correlation with osteoclast-like cell formation. Endocrinology 1999; 140 (8): 3552-61.

36. Locklin RM, Khosla S, Riggs BL. Mechanismo of biphasic anabolic and catabolic effects of parathyroid hormone (PTH) on bone cells. Bone 2001; 28 (Supl.) S80.

37. Khosla S. Minireview: the OPG/RANKL/RANK system. Endocrinology 2001; 142 (12): 5050-5.

38. Menaa C, Reddy SV, Kurihara N, Maeda H, Anderson D, Cundy T, et al. Enhanced RANK ligand expression and responsivity of bone marrow cells in Paget's disease of bone. J Clin Invest 2000; 105 (12): 1833-8.

39. Hughes AE, Ralston SH, Marken J, Bell C, MacPherson H, Wallace RG, et al. Mutations in TNFRSF11A, affecting the signal peptide of RANK, cause familial expansile osteolysis. Nat Genet 2000; 24 (1): 45-8.

40. Kong YY, Feige U, Sarosi I, Bolon B, Tafuri A, Morony S, et al. Activated $\mathrm{T}$ cells regulate bone loss and joint destruction in adjuvant arthritis through osteoprotegerin ligand. Nature 1999; 402 (6759): 304-9.

41. Itonaga I, Fujikawa Y, Sabokbar A, Murray DW, Athanasou NA. Rheur matoid arthritis synovial macrophage-osteoclast differentiation is osteoprotegerin ligand-dependent. J Pathol 2000; 192 (1): 97-104.

42. Jung K, Lein M, von Hosslin K, Brux B, Schnorr D, et al. Osteoprotegerin in serum as a novel marker of bone metastatic spread in prostate cancer. Clin Chem 2001; 47 (11): 2061-3.

43. Kong YY, Penninger JM. Molecular control of bone remodeling and osteoporosis. Exp Gerontol 2000; 35 (8): 947-56. 